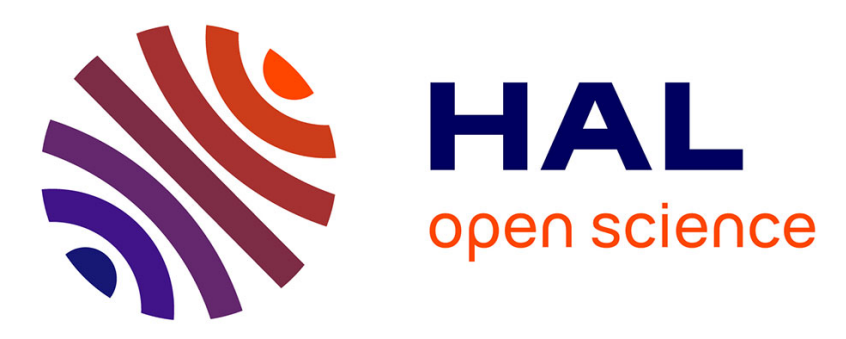

\title{
Notes sur deux sites de fours récemment découverts à Angkor
}

Christine Hawixbrock, Christophe Pottier

\section{To cite this version:}

Christine Hawixbrock, Christophe Pottier. Notes sur deux sites de fours récemment découverts à Angkor. Bulletin de l'Ecole française d'Extrême-Orient, 1996, 83 (1), pp.315 - 317. 10.3406/befeo.1996.3791 . halshs-02549036

\section{HAL Id: halshs-02549036 \\ https://shs.hal.science/halshs-02549036}

Submitted on 21 Apr 2020

HAL is a multi-disciplinary open access archive for the deposit and dissemination of scientific research documents, whether they are published or not. The documents may come from teaching and research institutions in France or abroad, or from public or private research centers.
L'archive ouverte pluridisciplinaire HAL, est destinée au dépôt et à la diffusion de documents scientifiques de niveau recherche, publiés ou non, émanant des établissements d'enseignement et de recherche français ou étrangers, des laboratoires publics ou privés. 


\section{Notes sur deux sites de fours récemment découverts à Angkor}

Christine Hawixbrock, Christophe Pottier

\section{Citer ce document / Cite this document :}

Hawixbrock Christine, Pottier Christophe. Notes sur deux sites de fours récemment découverts à Angkor . In: Bulletin de l'Ecole française d'Extrême-Orient. Tome 83, 1996. pp. 315-317;

doi : https://doi.org/10.3406/befeo.1996.3791

https://www.persee.fr/doc/befeo_0336-1519_1996_num_83_1_3791

Fichier pdf généré le 08/02/2019 


\section{CAMBODGE}

\section{Notes sur deux sites de fours récemment découverts à Angkor}

Au mois d'août 1995, deux sites inédits ont été signalés dans la région d'Angkor par l'équipe de l'université Sophia dirigée par M. Ishizawa, sur les indications de M.Ang Choulean, professeur à l'université des Beaux-Arts de Phnom Penh. Ces sites ont fait l'objet de plusieurs reconnaissances en collaboration avec nos collègues japonais et les services de la Conservation d'Angkor.

Le premier site, appelé Tuol Kpuos, est situé dans le village de Run Ta Ek, Srok Bantay Srei, à $3 \mathrm{~km}$ à l'est-nord-est du temple du Phnom Bok (coordonnées UTM 0393150 / 1489880). Il se présente comme un large tertre, sur lequel est installé le village repris par les troupes gouvernementales à la fin de 1994. La photodétection montre que le site n'est qu'un tronçon d'une « digue » discontinue orientée Nord-Sud, large d'environ $60 \mathrm{~m}$ et qui s'étend sur une longueur d'environ $3300 \mathrm{~m}$. Les renseignements recueillis sur place confirment cette analyse puisqu'ils mentionnent que le site archéologique s'étendrait sur plus d'un kilomètre (ce que nous n'avons pu vérifier, le site étant inaccessible plus au nord). On notera que la partie septentrionale de cette digue bloque le cours d'une petite rivière et la détourne le long de sa face orientale (fournissant ainsi l'eau nécessaire pour des potiers).

De nombreuses fouilles pirates avaient été réalisées avant nos premières reconnaissances. Elles avaient mis au jour une quantité très importante de tessons. À elle seule, cette quantité est déjà un indice de la présence de fours. Mais on peut aussi y observer des moutons (poteries déformées, soudées les unes aux autres...), des épernettes, des fragments de glaise portant des traces de modelage, d'autres provenant d'alandiers dont certains recouverts de glaçures. Il apparaît donc clairement que ce site correspond à un établissement de potiers khmers.

Une prospection sommaire du site et une première étude des objets collectés par la conservation et les services de la police du Patrimoine ont permis de noter quelques points:

- Les fours ont été utilisés pour une cuisson diversifiée allant d'une céramique de haute qualité à la production de tuiles.

- Globalement, on observe trois types de pâte: des grès fins et très sonores, de la terre cuite à pâte fine de couleur orange ainsi que des pâtes se rapprochant d'une porcelaine.

- Il semble qu'une nette majorité des tessons ne sont pas vernissés. La quantité restante non négligeable présente une glaçure verte (qui varie entre vert olive, vert acide et blanc). Nous n'avons noté ni glaçure brune, ni céramique chinoise.

- Les tuiles, dont une proportion appréciable présente une glaçure vert pâle, présentent de nombreux types (tuiles canal, tuiles couvre-joints, tuiles d'about, tuiles de noue et épis de faîtage). La brièveté des reconnaissances ne nous a pas permis de mesurer les tuiles courantes en vue d'identifier leur type. Cependant, on notera des tailles variables, en particulier pour les faîtages - essentiellement type « à selle» $\mathrm{A}$ ou $\mathrm{B}^{1}$. Les masques des tuiles d'about semblent 
pouvoir être rapprochés aux types B, C et E (Dumarçay, 1973, pl. XXXI et XXXII). Elles semblent donc devoir dater de la fin du IX' au début du XIe siècle.

- Les poteries présentent des formes très variées. On y a noté de nombreuses boites à couvercle de forme ronde (quelques-unes sont de forme cylindrique) pour la plupart glaçurées, des bouteilles (et des fragments de jarres) au col étroit et à la lèvre très évasées de taille très variable (diamètre entre $6 \mathrm{~cm}$ et plus de $30 \mathrm{~cm}$ ), de gros fragments de jarre sans col (peu glaçurés), peu de bols... Certains tessons présentent une épaisseur de pâte très fine. Continuer une énumération plus détaillée serait sans fondement vu le peu de temps passé sur le site (mais qui a quand même permis à la conservation d'Angkor et à la police de collecter plus de 200 pièces remarquables, essentiellement des boîtes). Il ressort cependant que ce site semble d'autant plus intéressant que la production semble être assez variée.

Dans l'état actuel de la céramologie khmère, notre datation s'est fondée sur les chronologies de B. P. Groslier et de R. Brown ${ }^{1}$. Elle coïncide approximativement à celle avancée avec les tuiles: du milieu du $\mathrm{IX}^{\mathrm{e}}$ au milieu du XI' ${ }^{\mathrm{e}}$ siècle.

Il est prématuré d'affirmer une date précise pour cet établissement. Cependant, cette brève analyse et quelques comparaisons avec des objets connus et datés suggèrent que cette partie du site a pu être en activité assez longuement, entre la fin du IX ${ }^{e}$ et le milieu du $\mathrm{XI}^{\mathrm{e}}$ siècle au moins. Notons que cette fourchette est assez logique si l'on analyse la localisation du site et les temples voisins.

Le second site, appelé «Vakan», est situé dans le Phum Bang Korg, Khum Ampil, Srok Bakong à 4,5 km au nord de la RN 6, le long de la route qui rejoint Lolei au Phnom Bok, récemment réalisée par le Bureau International du Travail (coordonnées UTM: 0390408 / 1479987). Il se présente comme un petit tertre de forme irrégulière, dominant le sol environnant d'environ $4 \mathrm{~m}$. La face orientale de ce tertre a été coupée par les terrassements de la route, mettant ainsi au jour une section de tertre intéressante. La photodétection montre que le site serait de forme carrée $(40 \times 40 \mathrm{~m})$, difficilement perceptible dans une topographie environnante assez mouvementée. On notera la présence du stung Roluos qui coule à $220 \mathrm{~m}$ à l'est, et un trapeang $(100 \times 50 \mathrm{~m})$ à la même distance à l'ouest-sud-ouest (non visité).

Le site n'a apparemment pas été pillé. Comme on l'a dit plus haut, seule sa partie orientale a été récemment rognée par la route. Cette coupe a mis au jour une quantité importante de tessons et de fragments de terre cuite (la surface du tertre présente, elle aussi, une forte concentration de tessons). On n'a pas noté de ratés de cuisson, mais des fragments de terre cuite portant des traces de cuisson et de vitrification pourraient indiquer que ce site correspond aussi à un établissement de potiers.

Un examen de surface du site, en particulier de la coupe sur la route, permet de noter quelques points:

- On observe trois types de pâte: du grès de qualité moyenne et des terres cuites orange et jaunes. La céramique est de qualité moyenne et commune sans aller cependant jusqu'à une céramique grossière.

- Parmi les nombreux tessons, nous n'avons pu trouver qu'un seul petit fragment de poterie glaçurée (vert olive sombre). Outre un fragment d'un bol moderne, nulle céramique chinoise n'a été trouvée. De nombreux tessons présentent une coloration en surface, un engobe chocolat sombre. Il nous a semblé pouvoir correspondre au lie-de-vin défini par B. P. Groslier (B. P. Groslier, 1981, p. 22; R. M. Brown, 1989, p. 44, Pl. 20a): teinte rouge sombre, violet ou parfois brun rouge.

- Le site ne présente pas de fragment de tuiles.

- Les poteries présentent des formes peu variées et jamais de petite taille: des jarres, avec ou sans col évasé, et des jarres à parois basses et larges ouvertures (appelées «cuvettes»

1. B. P. Groslier, "Introduction to the ceramic of Angkor ", in Khmers ceramics, 9th-14th Century, Southeast Asian Ceramic Society, Singapore, 1981. R. M. Brown, The Ceramics of SouthEast Asia, Their dating and identification, Oxford University Press, Singapore, 1989. 
dans H. Fujiwara, 1990, p. 2, fig. 1; et Brown, 1989, Pl. 25a) ${ }^{1}$. Certaines parties présentent des décors annulaires (bagues et motifs géométriques obtenus à l'aide d'une pointe).

- On note aussi l'absence complète de boîtes à couvercle de forme ronde ou cylindrique, de bouteilles, de bols et de poteries zoomorphes...

Il ressort que ce site semble correspondre à - au moins - un four khmer utilisé durant la période angkorienne. Les profils des tessons en sont assez caractéristiques. Le site ne présente ni tuile ni céramique «de luxe», objets habituellement aisés à dater. Il semble donc que nous ayons ici affaire à un petit four assurant une production assez commune (en opposition aux riches céramiques glaçurées ou aux «boîtes de cosmétiques» présentes dans le premier site). Cependant, les profils et les couleurs lie-de-vin semblent suggérer que ce four fut en activité à partir de la seconde moitié du $\mathrm{X}^{\mathrm{e}}$ siècle. Vu les types de poteries, on serait tenté de placer la fin de l'activité de ce four bien avant le XIIIe siècle.

Bien que l'ampleur de ce second site soit nettement plus réduite que celle du premier, sa production de céramiques présente un intérêt certain pour améliorer notre connaissance de ce type de céramique trop souvent négligé et qui reste une clef pour la datation des nombreux petits sites de fondations privées qui se répartissent dans la région d'Angkor.

On rappellera en conclusion que ce sont les premiers sites de fours découverts dans les environs immédiats d'Angkor. Leur découverte semble offrir des perspectives d'études très intéressantes qui permettraient de préciser la chronologie des céramiques à Angkor, instrument important pour les recherches archéologiques qui s'engagent actuellement.

Christine HAWIXBROCK et Christophe POTTIER

1. H. Fujiwara, Khmers ceramics from the Kamratan Collection, Oxford University Press, Singapore, 1990. 